\title{
Cell microarrays and RNA interference chip away at gene function
}

\author{
Douglas B Wheeler, Anne E Carpenter \& David M Sabatini
}

The recent development of cell microarrays offers the potential to accelerate high-throughput functional genetic studies. The widespread use of RNA interference (RNAi) has prompted several groups to fabricate RNAi cell microarrays that make possible discrete, in-parallel transfection with thousands of RNAi reagents on a microarray slide. Though still a budding technology, RNAi cell microarrays promise to increase the efficiency, economy and ease of genome-wide RNAi screens in metazoan cells.

With the genome sequences of dozens of species now complete, focus is shifting to the rapid elucidation of gene function. A number of highthroughput methods, such as protein-protein interaction assays, transcriptional profiling and gene sequence analyses, are used to infer gene function. Until recently, the more direct tools of classical genetics, for example gain- and loss-of-function mutants that yield insights into the molecular mechanisms of a cellular phenotype, have been difficult to implement at the genome-wide scale in cultured mammalian and Drosophila melanogaster cells.

Recent advances in RNAi are aiding the field of functional genomics by making loss-of-function genetic studies more tractable in numerous organisms. RNAi is a post-transcriptional method of gene silencing, originally discovered in Caenorhabditis elegans, in which double-stranded RNA (dsRNA) mediates the destruction of mRNAs in a sequence-specific fashion $^{1}$. Although the implementation of RNAi in different organisms varies, the capacity to silence genes selectively is bringing the power of loss-offunction genetics to systems not previously amenable to such studies.

There is a considerable push in the research community to generate genome-scale libraries of RNAi reagents for many organisms to allow broad screens in cultured cells ${ }^{2}$. Large cell-based RNAi screens are typically done in a 96- or 384-well plate format in which each well contains transfection reagent, cells and an RNAi reagent for a particular gene. Given the work involved in sample preparation and analysis, the automation of plate-based assays is vital to efficient screening. As such, laboratories with screening facilities use robots to dispense reagents and cells as well as automated plate readers or microscopes to analyze high-throughput experiments ${ }^{3,4}$.

Douglas B. Wheeler, Anne E. Carpenter and David M. Sabatini are at the Whitehead Institute for Biomedical Research and Massachusetts Institute of Technology Department of Biology, 9 Cambridge Center, Cambridge, Massachusetts 02142, USA. e-mail: sabatini@wi.mit.edu

Published online 26 May 2005; doi:10.1038/ng1560
Transfected cell microarrays are an advance in the miniaturization and simplification of high-throughput assays ${ }^{5}$. In this method, nucleic acids (expression plasmid or RNA) are printed on a standard glass microarray slide and used to transfect cells with the help of a transfection reagent ${ }^{5,6}$. The addition of cultured cells in medium to the array forms a living cell microarray of locally transfected cells in a lawn of nontransfected cells. Ziauddin and Sabatini dubbed this method 'reverse transfection' because cells are added on top of the nucleic acid instead of vice versa ${ }^{5}$.

Efforts by a number of groups to develop cell microarrays using RNAi have introduced the possibility of using cell microarrays for largescale RNAi studies ${ }^{7-15}$. Although this technique is still in its infancy, cell microarrays can considerably reduce the amount of effort necessary for rapid cell-based RNAi screens. Here we examine the current state of the field and the future of cell microarrays with an emphasis on their utility for high-throughput RNAi screens.

\section{Overexpression cell microarrays}

Microarray technology was developed in the early 1990s to measure the transcription levels of thousands of genes in parallel ${ }^{16,17}$. The basic premise of high-density arraying has since been expanded to create protein arrays for probing protein-protein or protein-small molecule interactions $s^{18,19}$, carbohydrate arrays $s^{20}$, tissue arrays ${ }^{21}$ and smallmolecule arrays ${ }^{22}$. In a twist of the canonical microarraying technique, the cell microarray, nucleic acids are printed in defined locations on a glass slide and used to transfect cells added on top of the microarray ${ }^{5}$.

Several groups have created cell microarrays that consist of arrayed spots of plasmid vectors or linear PCR products designed to express proteins of interest printed in an aqueous gelatin mixture ${ }^{5,23}$. A robotic microarrayer is used to print an array of nucleic acid spots on a glass slide (Fig. 1a). The printed array can be treated with transfection reagent before cells are added, or a stable transfection reagent may be printed along with the nucleic acid. Either way, the printed nucleic acid forms complexes with the transfection agent on the surface of the slide. The arrays are placed in tissue culture dishes, and cultured cells in medium are then added to the arrays in sufficient numbers so as to be near confluence upon termination of the experiment (Fig. 1b). Cells that adhere to the spots internalize the printed material and become transfected, and the remaining cells form a nontransfected lawn between spots. Depending on the transfection competency and the size of the cells being used, each feature of the array can consist of 30-500 transfected cells. At the end of an experiment, the microarrays are fixed and prepared for immunofluorescence, staining for DNA and F-actin, in 
a

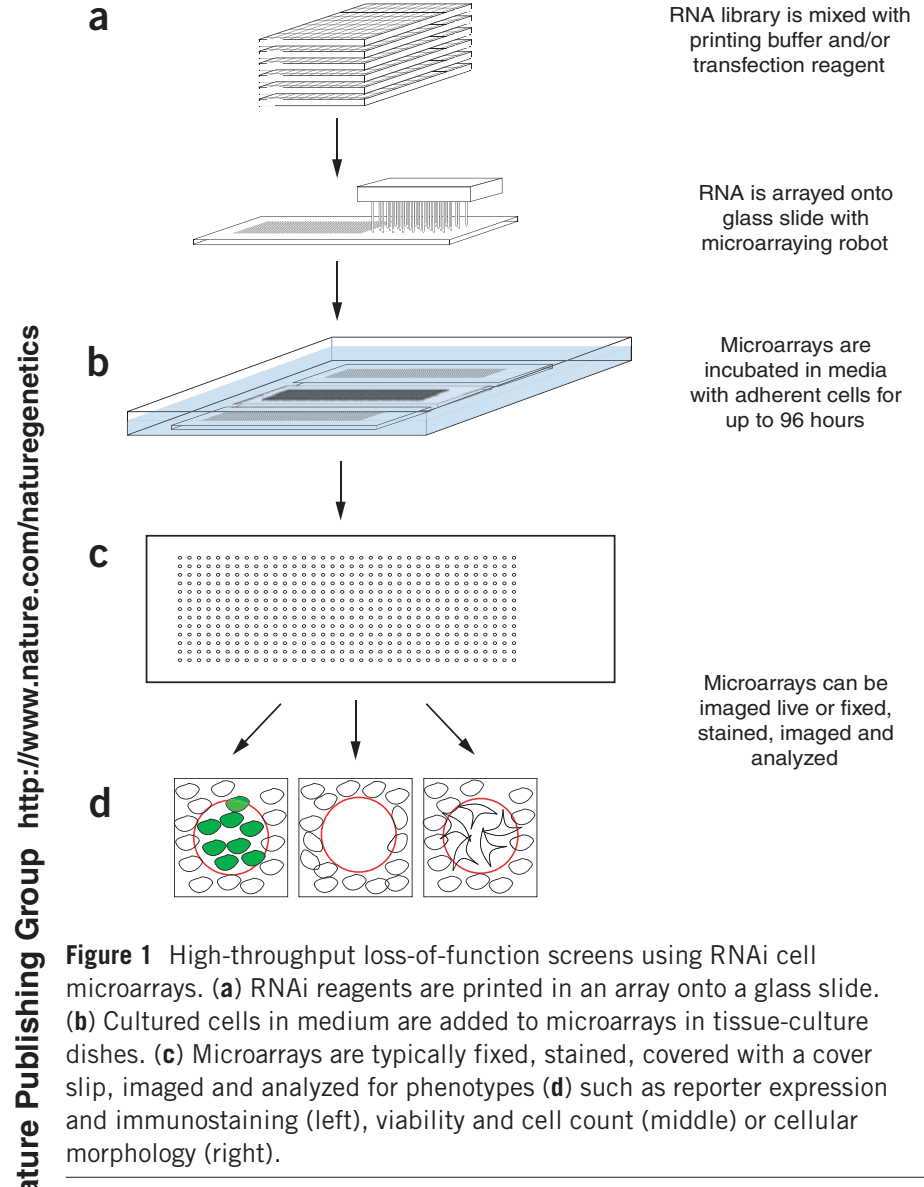

situ hybridization, apoptosis detection or other assays (Fig. 1c,d). The arrays are then covered with a cover slip and visualized with a microarray scanner or fluorescence microscope.

The utility of reverse transfection for high-throughput experiments was demonstrated by our laboratory in a proof-of-principle screen of 192 individual cDNAs in plasmid-expression vectors looking for genes whose overexpression provoked apoptosis, increased tyrosine phosphorylation or affected cell-cell adhesion in mammalian cells $s^{5}$. This study illustrated the compatibility of cell microarrays with assays such as immunofluorescence and TUNEL apoptosis detection and also demonstrated the binding of tritiated small molecules (FK506 and SCH23390) to overexpressed proteins (FKBP12 and DA receptor, respectively) by autoradiography.

Since this work was done, a handful of overexpression cell microarray studies have been published that offer technological improvements on the basic reverse transfection principle ${ }^{6,23-26}$. These studies have so far been limited to assay development or smaller screens. For example, pilot overexpression microarrays using GFP reporters were developed for the cAMP and serum response element pathways ${ }^{23,26}$. Examination of the subcellular localization of tagged proteins, which can help in inferring gene function, has also been demonstrated in microarray format ${ }^{24,25}$. With overexpression cell microarrays established as a method, a new focus in the cell microarray field has been the adaptation of cell arrays for RNAi in a high-throughput format.

\section{High-throughput RNAi screens in cultured cells}

Although protein overexpression is a valuable tool for examining gene function, it can result in nonphysiological phenotypes that may imply the wrong function for the gene of interest. As such, loss-of-function studies often provide a more direct method for functional studies. Until recently, gene-silencing studies were restricted to knockout strains of model organisms (yeast, mice, flies, worms, etc.), and antisense technology offered a limited avenue for transient gene-silencing in cultured cells $\mathrm{s}^{27}$.

The discovery that gene silencing at the post-transcriptional level can be mediated by dsRNA and small interfering RNAs (siRNAs) in organisms from nematodes to humans has advanced the field of functional genomics by allowing researchers to turn genes off. The selective 'knock-down' of genes has particular utility in the dissection of molecular pathways and the determination of epistatic relationships. Although the mechanism of RNAi is not yet fully understood, RNAi has become a standard instrument in the arsenal of the cellular biologist.

Implementation of RNAi varies depending on the organism of choice. For D. melanogaster or C. elegans, long dsRNAs homologous to the target mRNA can be used to induce RNAi ${ }^{1,28}$. The ribonuclease Dicer cleaves these dsRNAs into siRNAs of $\sim 21$ nucleotides to generate many siRNAs from each individual dsRNA. RNAi in mammals, however, is complicated by the interferon response, which is activated by exogenous dsRNAs longer than $\sim 30$ nucleotides ${ }^{29-31}$. Therefore, instead of using a long dsRNA that includes many 21-nucleotide sequences that can successfully initiate RNAi, individual 21-nucleotide siRNAs or short hairpin RNAs (shRNAs) must be designed and tested for each gene of interest. Despite rules that have been established to choose effective shRNAs and siRNAs, unknown secondary mRNA structure or factors render many RNAi constructs unable to initiate RNAi ${ }^{32}$. Currently, validated whole-genome collections of mammalian RNAi reagents with at least one effective RNAi construct per gene are not yet available.

Nevertheless, some groups have undertaken RNAi screening efforts in cultured human cells, demonstrating the utility of RNAi for the functional annotation of genes. Instead of generating large libraries of chemically synthesized siRNAs, several investigators have created their own large-scale shRNA or siRNA libraries encoded by plasmid vectors and used these libraries to identify new genes involved in the p53 pathway ${ }^{33}$, human proteasome function ${ }^{34}$ and the NF- $\kappa B$ signaling pathway ${ }^{35}$. These screens demonstrate the utility of large RNAi screens in mammalian cells but also underscore the need for better siRNA and shRNA selection methods because many RNAi constructs that should have elicited effects did not ${ }^{35}$. A development that may help with this issue is the use of endonuclease-prepared siRNAs, siRNAs that are generated by the RNase III-mediated degradation of long dsRNAs ${ }^{36}$. This process is an in vitro step that is analogous to the processing of dsRNAs into siRNAs in D. melanogaster cells and could ostensibly sidestep the problems of interferon response induction and siRNA specificity by providing many functional siRNAs to knockdown one gene. A library of 5,305 endonuclease-prepared siRNAs was recently used to identify genes not previously known to be involved in mitosis in HeLa cells ${ }^{37}$.

Finally, the ease of RNAi screening using dsRNA in D. melanogaster cells has resulted in several genome-wide screens done in a plate format ${ }^{4,38,39}$. Using cotransfected reporters, ATP production and cellular morphology as readouts, investigators have been able to assign gene function to several previously unannotated genes ${ }^{4,39-41}$. RNAi in cultured $D$. melanogaster cells using dsRNA rather than siRNA or shRNA has proven to be inexpensive, robust and usually free from the need for a transfection reagent owing to the phagocytic nature of many of the cell lines used ${ }^{28,39}$. In addition, the success rate of RNAi reagents in D. melanogaster cells is substantially higher than in mammalian cells because any one 600- to 800-bp dsRNA can be diced into many potentially effective $~ 21$-nucleotide siRNAs. D. melanogaster cells are well suited for genome-scale RNAi 
screens, particularly because comprehensive, validated siRNA or shRNA libraries do not yet exist for mammalian cells.

\section{RNAi cell microarrays}

Since 2003, several investigators have developed proof-of-principle RNAi cell microarrays using mammalian cells. One group described an RNAi reverse transfection method for murine and HeLa cells in a study that details the high-throughput design and validation of siRNA and shRNA probes ${ }^{7}$. Using eGFP fused to MyoD as a reporter for knockdown efficiency, the authors co-printed a MyoD-eGFP expression plasmid with transfection reagent and various siRNA and shRNA reagents targeting MyoD for knock-down in mammalian cells. The authors used this method to screen for the most effective siRNA candidates. This is particularly valuable because RNAi in mammalian cells relies on the careful selection and validation of siRNAs.

Mousses et al. developed a similar method with the goal of highthroughput RNAi experiments rather than siRNA probe selection ${ }^{13}$. They arrayed rhodamine-labeled siRNA targeting eGFP in a lipid transfection reagent onto glass slides and demonstrated potent and localized knock-down of eGFP expression in a lawn of HeLa cells stably expressing eGFP. This work was particularly important in showing localized effects of RNAi in the microarray format: in contrast to previous work, both the fluorescently tagged siRNA and its eGFP-silencing effects were shown not to spread outside each discrete spot. With molecules as small as siRNAs, proper sequestration of the construct in the printed spot is paramount to successful RNAi cell-microarray experiments. Finally, whereas most studies used RNAi to silence the expression of stable or cotransfected reporter genes, Erfle and colleagues showed the knock-down of endogenous $\beta C O P$ in HeLa cells on cell microarrays ${ }^{10}$. They were also able to assay a functional consequence of the knock-down of $\beta \mathrm{COP}$ by staining for a secretory marker whose transport to the cell surface was blocked when the expression of $\beta C O P$ was ablated, again demonstrating knock-down without the use of an exogenous reporter.

Primary and nonadherent cell lines have also been used on RNAi cell microarrays. Yoshikawa et al. achieved localized GFP knock-down in human mesenchymal stem cells on arrayed spots of transfection reagent, reporter plasmids, siRNAs and fibronectin ${ }^{9}$. Expanding the compatibility of cell microarrays to nonadherent and semiadherent cell lines, Kato et al. designed a biocompatible anchor for membrane surface to fabricate slides amenable to the attachment of nonadherent human erythroleukemic K562 cells ${ }^{11}$. These efforts are important in expanding cell microarrays to cell types that may seem intractable to this format.

Most published RNAi cell microarray methods are proof-ofconcept works that have yet to demonstrate parallel knock-down of multiple endogenous genes. These studies are limited by the difficulty of assembling a large number of siRNAs known to silence their target genes and the need for a sophisticated microscopy regimen to examine hundreds or thousands of spots of a cell microarray at high resolution. Overcoming these limitations has been most rapid in cultured D. melanogaster cells, for which the largest published RNAi cell microarray to date has covered 384 genes and used high-resolution automated image acquisition and analysis ${ }^{15}$.

In addition to screening each of these 384 gene knock-downs for cell proliferation, nuclear size and dAkt phosphorylation, we demonstrated the utility of $D$. melanogaster cell microarrays for simultaneous transfection of dsRNAs targeting two different genes. This allowed identification of synthetic phenotypes, as D. melanogaster cells treated with dPTEN dsRNA seeded onto an RNAi microarray showed elevated levels of phosphorylated dAkt, which could be suppressed or enhanced on spots of dsRNA targeting particular genes. Similar approaches will be a

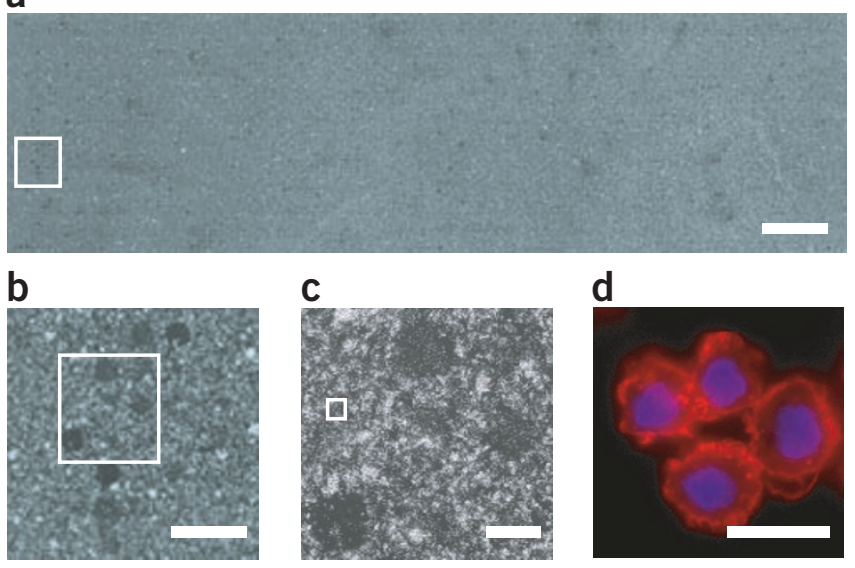

Figure 2 An example of a high-density, high-content RNAi cell-microarray screen in cultured D. melanogaster Kc167 cells. (a) A 'bird's-eye view' of a $D$. melanogaster RNAi cell microarray stained for cell nuclei. This array consists of 5,600 spots of distinct dsRNAs arrayed in a 40-row $\times 140$ column format on a standard $25 \mathrm{~mm} \times 75 \mathrm{~mm}$ glass slide. Low cell density 'hits' appear as dark spots devoid of nuclei and can be seen across the slide at this magnification. Scale bar, $5 \mathrm{~mm}$. (b) Higher-magnification image of the area in the white box from a shows concise spots where the nuclear density is decreased to varying degrees. Scale bar, $1 \mathrm{~mm}$. (c) Close-up of area in the white box from b shows cell-count hits. Scale bar, $250 \mu \mathrm{m}$. (d) For high-content phenotypes, images acquired in each printed spot of dsRNA are used to inspect cell morphology or quantify staining intensity. Scale bar, $20 \mu \mathrm{m}$. Blue, DNA; red, F-actin.

particularly useful for synthetic lethality experiments and for dissecting molecular pathways in D. melanogaster, as shown previously 38,39 .

Cell microarrays as a vehicle for high-throughput RNAi screens Expansion of RNAi cell microarrays to thousands of genes will allow for the conservation of RNAi reagents and cells and will greatly improve the speed of high-throughput studies with RNAi libraries. Given the apparatus necessary for screens in 96- or 384-well plates, high-throughput loss-of-function screening would be simplified by the use of cell microarrays for large RNAi experiments ${ }^{7-15}$. RNAi cell microarray slides should hold at least 5,000-6,000 spots of RNAi reagents, enabling whole-genome screens on a small number of slides ${ }^{8,15}$.

Figure 2 shows a prototype high-density RNAi cell microarray of D. melanogaster Kc167 cells (D.B.W. and D.M.S., unpublished data). This array consists of 40 rows and 140 columns of dsRNA spots to achieve a density of 5,600 distinct dsRNA spots per slide. dsRNA collections targeting most of the fly genome (including positive and negative controls) can be easily printed on four such slides. Inspection of this high-density array at a low magnification shows several viability phenotypes that present as localized dark spots of low cell density (Fig. 2a-c). Closer inspection of spots on the array with the help of high-resolution microscopy shows more subtle cellular phenotypes (Fig. 2d) and enables precise quantification of the phenotypes present (by immunostaining, DNA or actin staining, etc.).

The extension of RNAi cell microarrays to the full genomes of model organisms is promising. As cell microarrays become more widely used, so too will the tools for dealing with the large amount of quantitative data that can be collected from the thousands of individual RNAi experiments on one chip. The most salient issues that face cell microarray analysis are collection of high-content images of each cell population, automated analysis of each image and management of the resulting data. 
Figure 3 High-resolution image acquisition and analysis. (a) An automated microscope can be adapted to collect high-resolution images in each spot of the array, such that each image contains a population of cells with a particular gene knocked-down by RNAi. (b) These images can be viewed by eye or processed automatically with cell-image analysis software. A cluster of computers can speed this step. Tens or hundreds of phenotypes can be measured for every cell in every image, generating a large data set.

(c) Statistical and data-visualization methods for these multiparametric data are still in development. Phenotypes such as cell count (left column of left panel) yield a single number. To ease comparison of genes, phenotypes that are measured for each individual cell are reduced to a single number

\section{in a simple way (e.g., by calculating the mean or median) or in a more}

powerful way by statistically comparing histograms. Once the data have been reduced to a single number per phenotype per gene, they are treated like transcriptional microarray data, with each phenotype representing a different experiment. Thus, the data are normalized to controls and converted to a color reflecting an increase or decrease relative to controls for display in a red and green heat map (right panel). Clustering methods then identify families of genes whose knock-down produces similar cellular phenotypes.

\section{Imaging cell microarrays}

Without automation, analysis of a large cell-microarray experiment requires tens to hundreds of hours of microscopy. Increasingly, analysis of cell microarrays involves some amount of automation: usually high-

throughput image acquisition and often automated image analysis as well (Fig. 3). Two very different types of system are evolving to meet this need: microarray scanners that quickly provide a low-resolution providing a high-resolution readout. Selecting an appropriate system depends on the resolution required to visualize cellular phenotypes of interest ${ }^{42}$.

At one end of the spectrum are low-resolution assays for phenotypes such as cell growth or death or total amount of a protein detected by a specific fluorescently labeled antibody, where a glance at the entire spot relative to neighboring cells identifies the phenotype and individual cells need not be observed (Fig. 2b). In these cases, it may be feasible to acquire an image of the slide with a high-resolution microarray scanner, originally intended for use with traditional DNA microarrays ${ }^{8}$. In addition, some microarray scanners are being developed to acquire images at particularly high resolution so that individual cells can be distinguished ${ }^{43}$.

At the other end of the spectrum, high-resolution images are often required to visualize the phenotype of interest, for example subcellular localization (Fig. 2d). To automatically acquire images at high resolution, conventional microscopes have been outfitted with motorized components ${ }^{44}$ and have sometimes been purpose built for high-throughput imaging, often resembling plate readers more than traditional microscopes.

Unfortunately, all these microscope-derived instruments have been designed to acquire images from multiwell plates rather than cell microarrays, and so challenges remain in using them to image cell microarrays. For moderate-resolution phenotypes, it may be sufficient to capture slightly overlapping images across the entire slide. The images can then be compiled into a single large image of the entire slide, using software that often is packaged with the automated microscope $^{8}$. Some programs simply place images next to each other in the proper layout (yielding visible seams where images do not precisely align owing to the microscope stage's imprecision), whereas others use the cells at each juncture between images to automatically align the images more precisely. The resulting reconstructed image of the entire slide can then be cropped so that individual spots are analyzed independently.
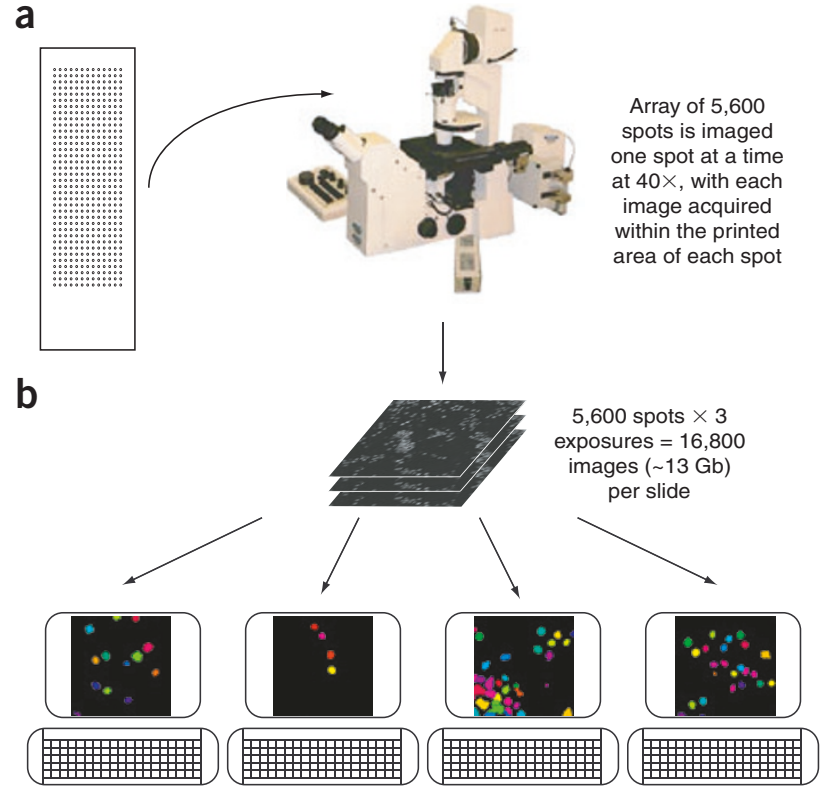

Batches of image sets are sent to clusters of computers for automated identification and measurement of individual cells

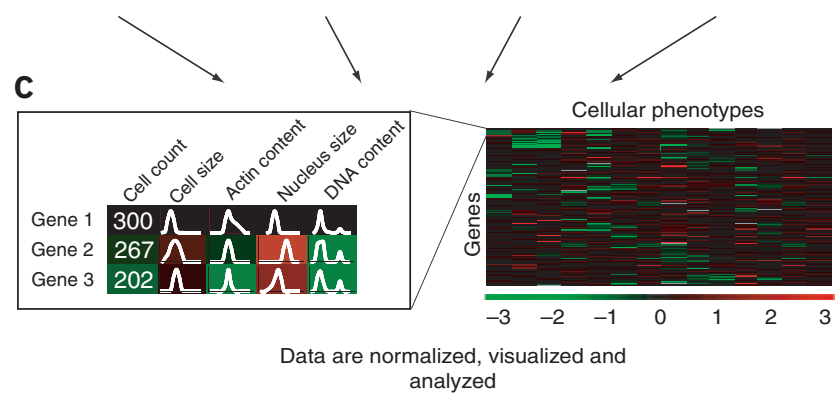

No commercially available systems have yet been designed for phenotypes requiring individual high-resolution images of each spot of a cell microarray. Though seemingly trivial, the small spot size relative to the wells of multiwell plates requires more accurate stage-positioning and software to compensate for slides placed slightly askew of the $x$ - $y$ axis of the microscope stage. With enough effort and patience, some existing software can be custom-adapted to collect high-resolution images from each spot of a cell microarray, allowing primary screens to be done with the data richness normally associated with secondary screens ${ }^{13,24}$.

\section{Image analysis}

For low-resolution phenotypes, once images have been acquired, visual inspection can determine which samples have produced phenotypes of interest. Yet, even when the visual output is obvious (e.g., a change in the intensity of staining of the spot), determining the identity of the reagent at each interesting spot can be tedious. Software tools are needed to make interaction with very large slide images more convenient (e.g., showing the sample names and grid coordinates for each spot and allowing the user to zoom in on spots of interest). Our laboratory is currently working to address this need (http://www.cellprofiler. org; A.E.C., T.R. Jones \& D.M.S., unpublished data).

Even for low-resolution phenotypes, automated image analysis is useful as it is quantitative and eliminates the tedium and bias of visual inspection. Automated image analysis is almost a necessity when highresolution images have been acquired for each spot of a cell microarray. It is possible to scan thousands of images by eye, looking for unusual 
phenotypes; for many complex phenotypes, there is not yet a substitute for the eye of an expert biologist ${ }^{39,40,42,45}$. Nevertheless, automated image analysis allows unbiased quantitative measurement of the shape, size, intensity and texture of cellular staining on a cell-by-cell basis and at much higher throughput. In addition, rather than simply identifying obvious, unusual cell samples as hits, measurements are made for every spot, which facilitates applications to systems biology ${ }^{2}$.

Although high-resolution images from each spot can be analyzed using existing software developed for multiwell plates, automated cellular image analysis is still an underdeveloped field of research. Excellent work has been done in this field in recent years ${ }^{46-48}$. Some researchers write custom software or macros for non-high-throughput image analysis programs, but their general applicability is limited ${ }^{8,13,24,40,42,49-57}$. A more user-friendly solution is proprietary commercial software, typically bundled with an expensive image acquisition instrument and targeted to the pharmaceutical drug screening market ${ }^{58}$ (for screening multiwell plates) or to clinical pathology laboratories ${ }^{59}$ (for histological slides). These platforms are well-suited to standard cell types in standard assays with very well defined visual outputs (e.g., cell count or nuclear/ cytoplasmic ratio), and the image analysis is usually rapid enough to keep pace with the image acquisition itself. This software typically does not have the flexibility required to adapt to nonmainstream phenotypes or nonstandard mammalian cell types, much less cells from nonmammalian organisms, which are particularly challenging to identify accurately ${ }^{15}$.

The high-throughput cell image analysis software project CellProfiler may solve many of these issues (http://www.cellprofiler.org/). This single, centralized package allows state-of-the-art advancements in image analysis research to be easily and rapidly plugged in and applied by biologists involved in high-throughput imaging. We are currently using this software to analyze high-resolution images from mammalian plate-based assays and D. melanogaster cell microarrays ${ }^{15}$.

The most pressing need in the field is continued improvement in the flexibility, accuracy and speed of image analysis. Automated image analysis for many phenotypes is currently intractable ${ }^{3}$, and the accuracy and robustness of the software across experimental conditions is limited, particularly for challenging cell types and phenotypes. For assays requiring intensive analysis, extracting multiple measurements (often hundreds ${ }^{53}$ ) can take several minutes on a standard computer, such that processing must be completed using clusters of computers. Methods for high-throughput analysis of three-dimensional image sets are also currently limited ${ }^{60}$. As cell microarrays become more routinely used, the automated acquisition and analysis of images from them should become more seamless ${ }^{24}$. Although 'on-the-fly' image analysis would give instant results, more sophisticated analysis requires that the entire image set be collected before full analysis. Therefore, integration of the two steps is not necessarily a pressing goal.

More important to the advancement of research in this field are protocols and tools for data storage and analysis ${ }^{61,62}$. Tools for organizing, visualizing and drawing conclusions from these large data sets are lacking. Transcriptional microarray data, with just one numerical data point per gene, are orders of magnitude simpler than the multidimensional phenotypic data from the automated image analysis of RNAi cell microarrays. Once processed, the images produce vast multiparametric data sets as each cell in an image is measured for many parameters across several fluorescent wavelengths. Currently, statistics and software to analyze the quantitative data coming out of large-scale screens are in a fledgling state and borrowed from other fields of study ${ }^{63}$. As the field of automated image analysis matures and draws more academic interest, the speed, accuracy and flexibility of analyzing images from cell microarrays should improve markedly, as will the tools for analyzing the resulting quantitative data.

\section{Concluding remarks and future directions}

RNAi cell microarrays offer an efficient platform for carrying out high-throughput loss-of-function studies. As RNAi collections become more complete and cell microarray technology progresses, sets of chips with genome-wide libraries of RNAi reagents should allow investigators to carry out and analyze large RNAi screens in days instead of weeks or months. The economy of RNAi cell microarrays will make possible the functional screening of whole genomes without investment in costly screening facilities. Should these tools become commercially available, researchers would only need access to appropriate tissue culture and imaging equipment to carry out rapid, large-scale RNAi screens. Furthermore, the miniaturization of RNAi screens into microarray format will allow the sparing use of RNAi reagents and rare cell lines. Currently, the key issues that must be resolved before RNAi cell microarrays become a routine tool are highresolution imaging platforms that are compatible with cell microarrays (which are currently available only as automated microscopes with custom software) and the availability of verified mammalian genome-wide RNAi libraries.

This platform may also evolve to accommodate assays on living cells in real time. Although several groups have demonstrated this capability with overexpression cell microarrays ${ }^{6,24}$, these experiments used arrays printed in the bottom of 12 -well plates or in chamber slides. Larger microscope slides fitted with cell-culture chambers will be needed to maximize the printable area on the slides in order to array large sets of RNAi reagents that can be analyzed in real time. High-content analysis of living RNAi cell microarrays poses a challenge, however, as the time required to image an entire slide of living cells at sufficient resolution may obscure transitory phenotypes of interest. Image analysis methods for time-lapse image sets are also in development ${ }^{64,65}$. These limitations are only technical, and overcoming them will make possible the realtime analysis of transient phenotypes such as subtle morphological changes or cellular calcium flux.

Perhaps the most practically important aspect of RNAi cell microarrays in need of development is the range of biological read-outs that can be used to infer function from large RNAi screens. For investigating signal-transduction pathways, many phosphorylation-specific antibodies exist that can be used for immunofluorescent read-outs on cell microarrays. When antibodies are not a tractable option (because they do not exist or because they are not compatible with immunofluorescence), the transient or stable expression of reporter constructs that express GFP or luciferase could allow the monitoring of gene expression or pathway activation ${ }^{26}$. Because groups often engineer such reporter cell lines for use on smaller assays, there should already be many fluorescent-based reporter lines that could be used on cell microarrays.

Finally, RNAi cell microarrays offer flexibility in carrying out combinatorial experiments in search of synthetic phenotypes. The treatment of cells with a drug or RNAi reagent targeting a gene of interest before adding them to an RNAi cell microarray will be very useful for epistatic analyses. Furthermore, screening for gene-small-molecule combinations or pairs of genes that yield synthetic lethality when knockeddown in concert could prove powerful in searching for cancer drug targets ${ }^{15,66}$. Conversely, cells could be transfected with overexpression constructs such that synthetic overexpression-knock-down combinations could be probed. The discovery of a gene that confers lethality when knocked-down only in cells that are overexpressing an oncogene could provide an inroad for new cancer therapies.

Although RNAi cell microarrays are a relatively young technology, they are capable of speeding up the pace of large RNAi experiments in cultured cells. As this platform matures, it will probably aid in the rapid, functional annotation of mammalian and D. melanogaster genomes. 


\section{ACKNOWLEDGMENTS}

We thank S.M. Ali, M.A. Domser, D.A. Guertin, J. Moffat and J.S. Van Arnam for comments and help during the preparation of this manuscript.

\section{COMPETING INTERESTS STATEMENT}

The authors declare that they have no competing financial interests.

Published online at http://www.nature.com/naturegenetics/

1. Fire, A. et al. Potent and specific genetic interference by double-stranded RNA in Caenorhabditis elegans. Nature 391, 806-811 (1998).

2. Carpenter, A.E. \& Sabatini, D.M. Systematic genome-wide screens of gene function. Nat. Rev. Genet. 5, 11-22 (2004).

3. Armknecht, S. et al. High-throughput RNA interference screens in Drosophila tissue culture cells. Methods Enzymol. 392, 55-73 (2005).

4. Boutros, M. et al. Genome-wide RNAi analysis of growth and viability in Drosophila cells. Science 303, 832-835 (2004).

5. Ziauddin, J. \& Sabatini, D.M. Microarrays of cells expressing defined cDNAs. Nature 411, 107-110 (2001).

6. Mishina, Y.M. et al. Multiplex GPCR assay in reverse transfection cell microarrays. J. Biomol. Screen. 9, 196-207 (2004)

7. Kumar, R., Conklin, D.S. \& Mittal, V. High-throughput selection of effective RNAi probes for gene silencing. Genome Res. 13, 2333-2340 (2003).

8. Baghdoyan, S. et al. Quantitative analysis of highly parallel transfection in cell microarrays. Nucleic Acids Res. 32, e77 (2004).

9. Yoshikawa, T. et al. Transfection microarray of human mesenchymal stem cells and on-chip siRNA gene knockdown. J. Control. Release 96, 227-232 (2004).

10. Erfle, H., Simpson, J.C., Bastiaens, P.I. \& Pepperkok, R. siRNA cell arrays for highcontent screening microscopy. Biotechniques 37, 454-458, 460, 462 (2004).

11. Kato, K., Umezawa, K., Miyake, M., Miyake, J. \& Nagamune, T. Transfection microarray of nonadherent cells on an oleyl poly(ethylene glycol) ether-modified glass slide. Biotechniques 37, 444-452 (2004).

12. Silva, J.M., Mizuno, H., Brady, A., Lucito, R. \& Hannon, G.J. RNA interference microarrays: high-throughput loss-of-function genetics in mammalian cells. Proc. Natl. Acad. Sci. USA 101, 6548-6552 (2004).

13. Mousses, S. et al. RNAi microarray analysis in cultured mammalian cells. Genome Res. 13, 2341-2347 (2003).

14. Chang, F.H. et al. Surfection: a new platform for transfected cell arrays. Nucleic Acids Res. 32, e33 (2004).

15. Wheeler, D.B. et al. RNAi living-cell microarrays for loss-of-function screens in Drosophila melanogaster cells. Nat. Methods 1, 127-132 (2004)

16. Fodor, S.P. et al. Light-directed, spatially addressable parallel chemical synthesis. Science 251, 767-773 (1991).

17. Schena, M., Shalon, D., Davis, R.W. \& Brown, P.O. Quantitative monitoring of gene expression patterns with a complementary DNA microarray. Science 270, 467-470 (1995).

18. Huang, J. et al. Finding new components of the target of rapamycin (TOR) signaling network through chemical genetics and proteome chips. Proc. Natl. Acad. Sci. USA 101, 16594-16599 (2004).

19. Zhu, H. et al. Analysis of yeast protein kinases using protein chips. Nat. Genet. 26 , 283-289 (2000).

. Houseman, B.T. \& Mrksich, M. Carbohydrate arrays for the evaluation of protein binding and enzymatic modification. Chem. Biol. 9, 443-454 (2002).

21. Kononen, J. et al. Tissue microarrays for high-throughput molecular profiling of tumor specimens. Nat. Med. 4, 844-847 (1998).

22. Kuruvilla, F.G., Shamji, A.F., Sternson, S.M., Hergenrother, P.J. \& Schreiber, S.L. Dissecting glucose signalling with diversity-oriented synthesis and small-molecule microarrays. Nature 416, 653-657 (2002).

23. Redmond, T.M. et al. Microarray transfection analysis of transcriptional regulation by cAMP-dependent protein kinase. Mol. Cell. Proteomics 3, 770-779 (2004).

24. Conrad, C. et al. Automatic identification of subcellular phenotypes on human cell arrays. Genome Res. 14, 1130-1136 (2004).

25. Palmer, E. \& Freeman, T. Investigation into the use of C- and N-terminal GFP fusion proteins for subcellular localization studies using reverse transfection microarrays. Comp. Funct. Genomics 5, 342-353 (2004).

26. Webb, B.L., Diaz, B., Martin, G.S. \& Lai, F. A reporter system for reverse transfection cell arrays. J. Biomol. Screen. 8, 620-623 (2003).

27. Kurreck, J. Antisense technologies. Improvement through novel chemical modifications. Eur. J. Biochem. 270, 1628-1644 (2003).

28. Clemens, J.C. et al. Use of double-stranded RNA interference in Drosophila cell lines to dissect signal transduction pathways. Proc. Natl. Acad. Sci. USA 97, 6499-6503 (2000).

29. Marcus, P.I. \& Sekellick, M.J. Interferon induction by viruses. XIII. Detection and assay of interferon induction-suppressing particles. Virology 142, 411-415(1985).

30. Elbashir, S.M. et al. Duplexes of 21-nucleotide RNAs mediate RNA interference in cultured mammalian cells. Nature 411, 494-498 (2001).

31. Dykxhoorn, D.M., Novina, C.D. \& Sharp, P.A. Killing the messenger: short RNAs that silence gene expression. Nat. Rev. Mol. Cell Biol. 4, 457-467 (2003).

32. McManus, M.T., Petersen, C.P., Haines, B.B., Chen, J. \& Sharp, P.A. Gene silencing using micro-RNA designed hairpins. RNA 8, 842-850 (2002).

33. Berns, K. et al. A large-scale RNAi screen in human cells identifies new components of the p53 pathway. Nature 428, 431-437 (2004).
34. Paddison, P.J. et al. A resource for large-scale RNA-interference-based screens in mammals. Nature 428, 427-431 (2004).

35. Zheng, L. et al. An approach to genomewide screens of expressed small interfering RNAs in mammalian cells. Proc. Natl. Acad. Sci. USA 101, 135-140 (2004).

36. Calegari, F., Haubensak, W., Yang, D., Huttner, W.B. \& Buchholz, F. Tissue-specific RNA interference in postimplantation mouse embryos with endoribonuclease-prepared short interfering RNA. Proc. Natl. Acad. Sci. USA 99, 14236-14240 (2002).

37. Kittler, R. et al. An endoribonuclease-prepared siRNA screen in human cells identifies genes essential for cell division. Nature 432, 1036-1040 (2004).

38. Lum, L. et al. Identification of Hedgehog pathway components by RNAi in Drosophila cultured cells. Science 299, 2039-2045 (2003).

39. Kiger, A. et al. A functional genomic analysis of cell morphology using RNA interference. J. Biol. 2, 27 (2003)

40. Eggert, U.S. et al. Parallel chemical genetic and genome-wide RNAi screens identify cytokinesis inhibitors and targets. PLoS Biol. 2, e379 (2004).

41. Bettencourt-Dias, M. et al. Genome-wide survey of protein kinases required for cell cycle progression. Nature 432, 980-987 (2004).

42. Yarrow, J.C., Perlman, Z.E., Westwood, N.J. \& Mitchison, T.J. A high-throughput cell migration assay using scratch wound healing, a comparison of image-based readout methods. BMC Biotechnol. 4, 21 (2004).

43. Ruckstuhl, T., Walser, A., Verdes, D. \& Seeger, S. Confocal reader for biochip screening and fluorescence microscopy. Biosens. Bioelectron. 20, 1872-1877 (2005).

44. Liebel, U. et al. A microscope-based screening platform for large-scale functional protein analysis in intact cells. FEBS Lett. 554, 394-398 (2003).

45. Huh, W.K. et al. Global analysis of protein localization in budding yeast. Nature 425 686-691 (2003).

46. Murphy, R.F., Boland, M.V. \& Velliste, M. Towards a systematics for protein subcelIular location: quantitative description of protein localization patterns and automated analysis of fluorescence microscope images. Proc. Int. Conf. Intell. Syst. Mol. Biol. 8, 251-259 (2000)

47. Murphy, R.F., Velliste, M. \& Porreca, G. Robust numerical features for description and classification of subcellular location patterns in fluorescence microscope images. J. VLSI Signal Process. 35, 311-321 (2003).

48. Eils, R. \& Athale, C. Computational imaging in cell biology. J. Cell. Biol. 161, 477-481 (2003).

49. Lindblad, J., Wahlby, C., Bengtsson, E. \& Zaltsman, A. Image analysis for automatic segmentation of cytoplasms and classification of Rac1 activation. Cytometry $A \mathbf{5 7}$ 22-33 (2004).

50. Mehes, G., Lorch, T. \& Ambros, P.F. Quantitative analysis of disseminated tumor cells in the bone marrow by automated fluorescence image analysis. Cytometry $\mathbf{4 2}$, 357-362 (2000).

51. Carpenter, A.E., Memedula, S., Plutz, M.J. \& Belmont, A.S. Common effects of acidic activators on large-scale chromatin structure and transcription. Mol. Cell. Biol. 25 , 958-968 (2005).

52. Carpenter, A.E., Ashouri, A. \& Belmont, A.S. Automated microscopy identifies estrogen receptor subdomains with large-scale chromatin structure unfolding activity. Cytometry A 58A, 157-166 (2004)

53. Perlman, Z.E. et al. Multidimensional drug profiling by automated microscopy. Science 306, 1194-1198 (2004).

54. Perlman, Z.E., Mitchison, T.J. \& Mayer, T.U. High-content screening and profiling of drug activity in an automated centrosome-duplication assay. Chembiochemistry 6 145-151 (2005).

55. Obenauer-Kutner, L.J. et al. Use of an automated image processing program to quantify recombinant adenovirus particles. Microsc. Microanal. 11, 37-41 (2005).

56. Camp, R.L., Chung, G.G. \& Rimm, D.L. Automated subcellular localization and quantification of protein expression in tissue microarrays. Nat. Med. 8, 1323-1327 (2002).

57. Abramoff, M.D., Magalhaes, P.J. \& Ram, S.J. Image processing with ImageJ. Biophotonics Int. 11, 36-42 (2004).

58. Ghosh, R.N., Grove, L. \& Lapets, O. A quantitative cell-based high-content screening assay for the epidermal growth factor receptor-specific activation of mitogen-activated protein kinase. Assay Drug. Dev. Technol. 2, 473-481 (2004).

59. Oode, K. et al. The development of a cell array and its combination with laser-scanning cytometry allows a high-throughput analysis of nuclear DNA content. Am. J. Pathol. 157, 723-728 (2000)

60. Belien, J.A. et al. Confocal DNA cytometry: a contour-based segmentation algorithm for automated three-dimensional image segmentation. Cytometry 49, 12-21 (2002).

61. Searls, D.B. Data integration: challenges for drug discovery. Nat. Rev. Drug Discov. 4 , 45-58 (2005).

62. Swedlow, J.R., Goldberg, I., Brauner, E. \& Sorger, P.K. Informatics and quantitative analysis in biological imaging. Science 300, 100-102 (2003).

63. Swayne, D.F., Buja, A. \& Temple-Lang, D. Exploratory visual analysis of graphs. Proceedings of the Third Annual Workshop on Distributed Statistical Computing (Technische Universität Wien, Vienna, 2003).

64. Cheezum, M.K., Walker, W.F. \& Guilford, W.H. Quantitative comparison of algorithms for tracking single fluorescent particles. Biophys. J. 81, 2378-2388 (2001).

65. Bacher, C.P., Reichenzeller, M., Athale, C., Herrmann, H. \& Eils, R. 4-D single particle tracking of synthetic and proteinaceous microspheres reveals preferential movement of nuclear particles along chromatin-poor tracks. BMC Cell. Biol. 5, 45 (2004).

66. Hartwell, L.H., Szankasi, P., Roberts, C.J., Murray, A.W. \& Friend, S.H. Integrating genetic approaches into the discovery of anticancer drugs. Science 278, 1064-1068 (1997). 